\title{
Phubbing y género en un sector académico en Quito: Uso, abuso e interferencia de la tecnología
}

\author{
Phubbing and Gender in an Academic Sector in \\ Quito: Use, Abuse and Interference of Technology
}

\author{
Cristian Moisés Villafuerte-Garzón (®) https://orcid.org/0000-0002-3054-7272 \\ Instituto Tecnológico Superior Cordillera, Ecuador, cristian.villafuerte@cordillera.edu.ec \\ María Vera-Perea (D) https://orcid.org/0000-0003-2449-4685 \\ Universidad Especialidades Espiritu Santo,Ecuador,mveraperea@uees.edu.ec
}

Recepción:

$06 / 11 / 2017$

Aprobación:

$28 / 08 / 2018$

\begin{abstract}
The objective of the present study is to explain the interference of technology in daily life, performing a differential study by gender. The specific objectives are to ascertain if there are significant differences between men and women as regards periods of time, reasons for using technology, technological devices used, social problems that arise from the interference of technological devices in different aspects of life, time that is possible to spend without technology, feelings of guilt and perception of addiction. In order to accomplish these objectives, 3,793 people were surveyed. The sample is made up of students, academic, administrative and service staff from an academic institution located in Quito (Ecuador). Descriptive analyses, $t$ student test and chi squared were performed. It has been demonstrated that there are addictive behaviors to information and communication technologies, as well as the presence of phubbing in the surveyed population. Moreover, significant differences between genders were verified.
\end{abstract}

Key words: tech addiction, technology, gender, phubbing.

Resumen: El artículo tiene como objetivo explicar la interferencia de la tecnología en la vida cotidiana, con un estudio diferencial por género. Los objetivos específicos son conocer si existen diferencias significativas entre hombres y mujeres en: periodos; razones que les impulsan a usar la tecnología; dispositivos tecnológicos utilizados; problemas sociales que se derivan de la interferencia de los dispositivos tecnológicos en diferentes aspectos de la vida; tiempo que pueden soportar sin utilizar tecnología; sentimiento de culpabilidad; y percepción de adicción. Se encuestaron 3,793 personas, entre estudiantes, personal académico, administrativo y de servicios de una institución académica situada en Quito, Ecuador. Mediante análisis descriptivos, pruebas $t$ de Student y chi cuadrado, se ha logrado establecer un hito referencial, al demostrar que existen comportamientos adictivos a las tecnologías de información y comunicación (TIC), así como la presencia de phubbing en la población encuestada, habiendo diferencias significativas por género.

Palabras clave: tecnoadicción, tecnología, género, phubbing. 


\section{Introducción}

Vivimos en un mundo lleno de dispositivos tecnológicos inteligentes, donde se consume información bajo demanda. Se han creado millones de formatos de conexión e interactividad que marcan el ritmo de uso, consumo y abuso de las tecnologías de la información y comunicación (TIC). De hecho, según Sanou (2016: 4), a finales de 2016, alrededor del 47\% de la población mundial tenía conexión a Internet - de la cual el $44.9 \%$ son mujeres y el $51.1 \%$ son hombres-, hay 7.1 billones de suscriptores y suscriptoras a líneas celulares, y más de 900 millones de redes sociales y sistemas de comunicación instantánea, lo cual muestra la gran cantidad de información producida y demandada por estos usuarios/as.

En los estudios sobre el uso de tecnología, normalmente se tiende a pensar en el/la usuario/a como un ser universal, sin ser diferenciado por género, y la mayoría de los productos y contenidos digitales también están diseñados de la misma manera, dejando de lado características importantes que diferencian a hombres y mujeres; lo mismo pasa al hablar de phubbing ${ }^{1}$ o smombie. ${ }^{2}$ Por tanto, el objetivo general de este artículo será analizar el uso y abuso de la tecnología y su interferencia, analizando conductas de adicción y phubbing, teniendo en cuenta el rol de género.

Los dispositivos tecnológicos de uso diario fueron desarrollados, en principio, para hacer más fácil la vida. La computadora, el smartphone y las tablets se han vuelto parte de la cotidianidad; pero estos dispositivos no solo revolucionaron el ritmo de vida, sino a la sociedad, permitiendo nuevas formas de conexión y socialización entre las personas (Castells, 1996: 405). Casi sin darnos cuenta han interferido en todas las actividades, obligando a vivir cada instante a través de ellos, creando, mezclando, produciendo y posteando más información de la que se podría consumir en actividades de trabajo, entretenimiento, comunicación, salud y otras; por no decir que en todas se incluye uno o varios dispositivos tecnológicos, al punto de cambiar desde la forma de comunicarse, hacer negocios o prestar servicios, hasta las prioridades económicas.

En cuanto al uso y consumo de tecnología relacionados con el género, tradicionalmente se habla de un patriarcado de dominación masculina en torno a las TIC (Sanz, 2006: 194); aunque hombres y mujeres siempre han

1 Phubbing proviene de la combinación de las palabras en inglés Phone (teléfono) y Snub (ignorar).

2 Smombie proviene de la combinación de las palabras smartphone (teléfono inteligente) y zombi. 
trabajado juntos en el desarrollo de soluciones tecnológicas de cada época. Por ejemplo, si Ada Lovelace no hubiera conocido a Babbage, hoy seguramente no se disfrutaría de la computación como la conocemos; o si en el desarrollo de la ENIAC no hubieran intervenido seis mujeres que rompieron los esquemas tradicionales de esa época, se hubiera llevado más tiempo desarrollar la computadora personal que ahora se tiene. Las mujeres crean tecnología que mueve al mundo (Isaacson, 2014: 53).

Pero en realidad, la participación igualitaria de hombres y mujeres se ha visto opacada en la construcción, desarrollo, uso e innovación de las TIC, generando discriminación en esta área. En 1992, la Unión Europea alertó sobre la baja participación de las mujeres en la sociedad de la información; lo mismo sucedió en Beijing, en 1995, cuando en la Conferencia Mundial de las Naciones Unidas sobre la Mujer se indicó que las TIC eran el sector más crucial para lograr igualdad, democracia y justicia social (Naciones Unidas, 1996). Para Cantos (2017: 50), la baja representación de mujeres en las profesiones relacionadas con las TIC es un fenómeno generalizado. Asimismo, su nivel de habilidades digitales - esenciales en el actual contexto socioeconómico- es, en muchas ocasiones, relativamente inferior al de los hombres.

En su investigación, Rodríguez (2009: 4) señala esto como una brecha digital de género, ya que desde su visión hay barreras para el acceso y uso de las TIC, no solo porque existe un menor número de usuarias, sino por las persistentes desigualdades estructurales; por ejemplo, producción de información y contenidos sesgados que limitan el acceso de las mujeres al consumo de contenidos digitales. Además, esta brecha también es resultado de condicionamientos culturales (Rodríguez, 2009: 11), prejuicios y estereotipos, que producen una estratificación por género.

La difusión de las nuevas TIC no es neutral respecto al género, y sus efectos variarán en función del origen de la mujer, de su clase social, posibilidad de acceso a la educación o incluso raza o edad (Castaño et al., 2010: 87). Términos como tecnofeminismo y ciberfeminismo, entre otros, han sido introducidos para explicar el aporte de género, identidades y sexualidades en las TIC, a fin de conseguir espacios de participación con visiones no tan sesgadas. Sin embargo, las diferencias de género todavía existen en el uso de determinados tipos de tecnología. La parte más visible del ciberfeminismo se ha centrado básicamente en explorar las posibilidades que ofrecen las TIC para la deconstrucción de los géneros (Reverter, 2013: 455).

Cotten et al. (2009: 1180) encontraron que fueron pocas las diferencias de género en el uso de dispositivos móviles para actividades de comunicación; 
además, identificaron que los hombres ocupaban más los teléfonos móviles para fines recreativos como juegos, fotos y video. Por su parte, Hargittai y Walejko (2008: 252) demostraron que aunque había igualdad de género respecto al acceso a las TIC, ciertas actividades, como el uso compartido de contenido digital en línea, todavía mostraban marcadas diferencias de género, al haber más contenidos compartidos por hombres que por mujeres. Estas diferencias desaparecieron cuando se tomaba en cuenta la interactividad del usuario/a de la web. Finalmente, Rideout et al. (2010: 21) identificaron que los niños (entre 8 y 18 años) pasaban más tiempo utilizando ordenadores que las niñas, pero gran parte de ese periodo los niños lo dedicaban a los videojuegos; en cuanto a las niñas, ocupaban más tiempo en sitios de redes sociales, escuchando música o haciendo lectura en línea.

Lo que parece evidente, es que el ciberespacio reproduce en gran medida los estereotipos culturales de género (Sanz, 2006: 201). Según Ensmenger (2010: 2), el campo de la informática está dominado por hombres y asociado con atributos masculinos, supuestamente por ser muy técnico, y por requerir largas horas de trabajo y ser seres antisociales. En su investigación sobre hábitos y experiencias de consumo tecnológico de tweens ${ }^{3}$ guayaquileños (Ecuador) por género, Gómez (2017: 71) indica que las mujeres son más pragmáticas que los varones en temas de tecnología; asimismo la literatura revela que los juegos de consolas tienen naturaleza masculina. Por lo tanto, los varones ocupan más horas jugando videojuegos, y las mujeres prefieren las redes sociales.

Todo lo anterior nos lleva al planteamiento de las siguientes hipótesis:

$\mathrm{H}_{1}$ : Los hombres usan los dispositivos tecnológicos por periodos más prolongados que las mujeres de la muestra analizada.

$\mathrm{H}_{2}$ : Las razones que impulsan a hombres y mujeres a utilizar dispositivos tecnológicos son diferentes en la muestra analizada. Dichas razones son: búsqueda de información, compras, estudios, redes sociales, reproducción de videos, juegos en red, ocio, servicios de comunicación y trabajo.

$\mathrm{H}_{3}$ : Los dispositivos utilizados por hombres y mujeres para cada uno de los usos son distintos en la muestra analizada. Dichos dispositivos son: PC, smarphone, tablet y consola de juegos.

3 Se trata de la población infantil de 8 a 13 años. El término tween proviene de la palabra inglesa between (entre). 
Al escribir sobre la sociabilidad virtual, Cáceres et al. (2017: 5) expresaron que la mediación de la tecnología elimina la necesidad de acceso físico y directo con el otro en la comunicación, y transforma los aspectos psicosociales que quedan comprometidos en la interacción presencial. Por tanto, quien no interactúa a través de un dispositivo tecnológico con otros en la red no existe y podría considerarse como un marginado, por el simple hecho de no estar conectado, lo cual ya evidencia problemas por el uso excesivo de dispositivos tecnológicos.

Y ya que las nuevas TIC han cambiado el ritmo de vida y se han convertido en elementos esenciales del diario vivir, más allá de ser solo dispositivos o extensiones corpóreas para realizar casi toda actividad, los estudios referentes a tiempos de uso prolongados y reiterados han establecido hitos referenciales de adicción, determinando, por ejemplo, que la adicción a las nuevas tecnologías se caracteriza por un consumo abusivo del ordenador, Internet, móvil, videojuegos y redes sociales. Y aunque las adicciones pueden afectar a todas las edades, Rodríguez et al. (2012:355) demuestran que en los adolescentes existe un índice más alto que en los de mayor edad. Cabe señalar que estos estudios normalmente se dirigen a establecer si existe la adicción y su sintomatología.

A partir de investigaciones previas sobre las actividades, tiempos y periodos de uso de dispositivos tecnológicos, se observa la interferencia que estos últimos generan en la vida cotidiana, catalizando problemas sociales de adicción a las nuevas TIC.

Uno de estos problemas es el phubbing: ignorar a las personas que están alrededor por prestar más atención a los dispositivos tecnológicos. Según Treviño (2013: 1), el phubbing consiste en el acto de menospreciar a quien nos acompaña por prestarle mayor atención a un aparato electrónico que a la persona, es decir, se ignora a quien se tiene enfrente por utilizar el teléfono móvil.

Yendo un paso más allá, el phubbing es el verdadero problema de las parejas actuales (Roberts y David, 2016: 140). La página stopphubbing.com (Haigh, 2013) informa cómo en restaurantes se observan, en promedio, 36 casos de phubbing por cena; esto es, pasar solos mientras se está en compañía de los demás. Asimismo, el $87 \%$ de los/as adolescentes prefiere comunicarse a través de texto que cara a cara; y es por algunas de estas razones que el phubbing ha sido etiquetado como "el fin de la civilización" (Haigh, 2013: 1). Sin llegar a esos extremos, parece que los cambios conductuales por la interferencia de los dispositivos tecnológicos podrían ser la causa de estos nuevos problemas sociales. González et al. (2018: 90) revelaron que el 
phubbing impacta negativamente en la satisfacción dentro de las relaciones románticas y la salud mental de los individuos. Aquellos que tuvieron más características de phubbers también mostraban mayor sintomatología asociada con la depresión, la ansiedad y el estrés, menor satisfacción en la relación de pareja y menos bienestar psicológico.

Los dispositivos tecnológicos como el smartphone mantienen al tanto a las personas de todo lo que sucede en cada instante, sea información importante o no, necesaria o no interesante; en dichos dispositivos se reciben constantemente notificaciones de lo que está ocurriendo en la vida digital de la gente. Estudios previos demuestran que la tecnología interfiere en la vida cotidiana de las personas. Roberts y David (2016: 139) expusieron un ejemplo donde la mayoría de las parejas analizadas reconoció haberse visto afectada alguna vez por la presencia activa de un teléfono celular en una escena íntima. Los especialistas también indicaron que el phubbing conduce a trastornos como la depresión y la ansiedad.

En la actualidad no hay estudios sobre phubbing y género en comunidades académicas en Quito, Ecuador. Para cubrir ese vacío, se plantean las siguientes hipótesis:

$\mathrm{H}_{4}$ : Los problemas sociales que se derivan de la interferencia de los dispositivos tecnológicos en diferentes aspectos de la vida son distintos en el caso de hombres y en el de mujeres de la muestra analizada.

$\mathrm{H}_{5}$ : Hombres y mujeres admiten poder soportar distintos tiempos sin utilizar tecnología en la muestra analizada.

$\mathrm{H}_{6}$ : Existen diferencias significativas en cuanto al sentimiento de culpabilidad en hombres y mujeres de la muestra analizada por pasar largos periodos utilizando dispositivos tecnológicos como la computadora personal, smartphone, tablet y las consolas de juego.

$\mathrm{H}_{7}$ : Hombres y mujeres de la muestra analizada reportan diferentes percepciones de adicción a la tecnología.

\section{Método}

\section{Muestra y procedimiento}

Se llevó a cabo un muestreo por conveniencia en una institución educativa de educación superior ubicada en la ciudad de Quito, Ecuador, a todo su personal (estudiantes, docentes, personal administrativo y de servicios). El cuestionario se 
aplicó a través de Internet, con dos modalidades. En primer lugar, se utilizaron los laboratorios de la institución, para que los estudiantes y el personal de servicios pudieran acceder y completar el cuestionario. En segundo lugar, al personal docente y administrativo se les envió la dirección de publicación de la encuesta, vía correo electrónico, para que la contestaran. El cuestionario se mantuvo activo durante dos semanas.

La muestra está formada por 3,793 participantes (58\% de la población total), de los cuales $60.3 \%$ son mujeres y $39.7 \%$, hombres. La edad media es de 23 años (DT $=0.5)$. El 90\% de la muestra está en proceso de cursar estudios de tecnología, el 5.6\% terminó bachillerato, 1.1\% son tecnólogos, $1.1 \%$ son ingenieros, $0.9 \%$ finalizó su maestría, $0.6 \%$ está estudiando una ingeniería, $0.4 \%$ está cursando una maestría, $0.1 \%$ no tiene estudios, $0.1 \%$ tiene estudios de primaria, $0.1 \%$ está realizando un $\mathrm{PhD}$ y $0.1 \%$ es $\mathrm{PhD}$. En cuanto al trabajo, $44.6 \%$ no tiene empleo, $10.5 \%$ labora en empresas públicas, y $44.9 \%$ trabaja en empresas privadas.

\section{Instrumentos}

Se creó una batería completa de preguntas acerca de la tecnología para dar respuesta a todas las hipótesis del estudio. Por tanto, todos los ítems son autocreados, con el fin de responder a dichas hipótesis.

Periodicidad de uso. Se midió la frecuencia de utilización para cada uno de los dispositivos (PC, smartphone, tablet y consola de juegos), con una respuesta tipo Likert de 9 puntos (desde 1=Nunca; hasta 9=Más de 15 horas diarias).

Impulso al uso de tecnología. Los participantes debían escoger una opción sobre qué les impulsa a ocupar la tecnología de entre las siguientes: uso de redes sociales, trabajo, estudios, estar conectado con la familia, moda, sociedad y ocio.

Utilización de los dispositivos. Los participantes debían marcar el porcentaje del tiempo que dedican en una tabla de doble entrada: la primera indicaba el tipo de dispositivo, y la segunda, la actividad. Las actividades fueron: buscar información, hacer compras en línea, cursar estudios regulares, estar en redes sociales, reproducir videos, cursar autoeducación, jugar en red, tener ratos de ocio, de servicios de comunicación, y de trabajo.

Problemas derivados de un uso excesivo de la tecnología. Los participantes debían señalar en una escala Likert de 5 puntos (desde $1=$ Nunca; hasta $5=$ Siempre), la frecuencia con la cual habían tenido problemas por ocupar de manera excesiva la tecnología en las siguientes áreas: en casa o con la familia, 
en el trabajo, en el lugar de estudios, en medios de transporte, haciendo deporte, en centros comerciales o tiendas y en reuniones sociales.

Tiempo sin utilizar la tecnología. Se midió la frecuencia de cuánto tiempo pueden pasar los participantes sin ocupar cada uno de los dispositivos, con una respuesta tipo Likert de 10 puntos (desde $1=$ Nunca; hasta 10=Todo un día sin problemas).

Culpa. Los participantes debían señalar en una escala Likert de 6 puntos (desde $1=$ Frecuentemente; hasta $6=$ Nunca) si sentían culpa por estar largo tiempo utilizando cada uno de los cuatro dispositivos tecnológicos.

Adicción a la tecnología. Para medir este constructo, los participantes contestaban dos ítems En primer lugar, se les preguntaba directamente si, en su opinión, creían que sufrían una adicción a la tecnología, a lo cual debían responder de manera dicotómica. En segundo lugar, se les preguntaba si habían regresado alguna vez a casa, trabajo o lugar de estudios por haber olvidado su dispositivo móvil sin importar que estuvieran atrasados; aquí también debían contestar de manera dicotómica.

\section{Análisis de datos}

Los análisis de datos para poner a prueba las hipótesis de estudio fueron los siguientes: en primer lugar, los descriptivos, y en segundo, las pruebas $t$ de Student y de chi cuadrado.

\section{Resultados}

Para someter a prueba la $\mathrm{H}_{1}$ y verificar si hombres y mujeres poseen una periodicidad de uso de los diferentes dispositivos tecnológicos, se llevó a cabo una prueba $t$ de Student para muestras independientes, con lo cual se obtuvieron diferencias significativas, al ser siempre los valores más elevados y, por tanto, más horas de uso de los dispositivos reportados en el caso de los hombres (véase Tabla $1^{4}$ ). Con ello se puede confirmar la $\mathrm{H}_{1}$ : los hombres reportan un mayor uso de todos los dispositivos tecnológicos.

En el caso de la $\mathrm{H}_{2}$, se realizaron dos tipos de pruebas: en primer lugar un chi cuadrado para analizar si hay diferencias en cuanto a hombres y mujeres cuando marcan qué les impulsa a utilizar la tecnología (las opciones fueron: uso de networking/redes sociales, trabajo, estudios, estar conectado con la familia, moda, sociedad y ocio) y el resultado fue de $85.61(\mathrm{p}=.000)$. 4 Todas las tablas y figuras se encuentran en el Anexo, al final del presente artículo (Nota del editor). 
En segundo lugar, se efectuó una prueba $t$ de Student para muestras independientes, donde se observaron diferencias significativas respecto a las horas dedicadas al día en los siguientes aspectos: compras on line, redes sociales, reproducción de videos, juegos en red, ocio, servicios de comunicación y trabajo. Se encontró que los hombres dedican más tiempo a estas actividades en el día. En cuanto a la búsqueda de información y los estudios, ambos géneros no mostraron diferencias significativas (véase Tabla 1). Dichas diferencias se ven muy claramente en la Figura 1.

$\mathrm{La} \mathrm{H}_{3}$ está muy relacionada con la anterior, pero en cuanto al dispositivo utilizado para cada uso. En este caso, los datos han sido divididos por dispositivos. Como se puede observar en la Tabla 1, para todos los usos y en todos los dispositivos hay diferencias significativas por género (excepto en la búsqueda de información con PC y SP); en el caso de los hombres, el porcentaje es siempre más elevado.

Respecto a la $\mathrm{H}_{4}$, que aborda los problemas de interferencia que pueden ocurrir por el uso de la tecnología, los resultados de la prueba $t$ de Student para muestras independientes (Tabla 1) señalan cómo, efectivamente, existen diferencias importantes en cuanto al género, y siempre son más elevadas en el caso de los hombres. Asimismo, al observar la Figura 4 se puede notar cómo se presentan problemas por la interferencia de los dispositivos tecnológicos sin importar el lugar. En todos los casos, hombres y mujeres mantienen una tendencia similar de respuesta.

Respecto a la $\mathrm{H}_{5}$-sobre la cantidad de tiempo que hombres y mujeres pueden pasar sin utilizar sus dispositivos tecnológicos-, es importante destacar que cuanta más elevada sea la puntuación, significa que menos problema tienen para estar sin sus dispositivos. Los resultados de la prueba $t$ de Student para muestras independientes (Tabla 1) indican que la única diferencia significativa se da en el caso del tiempo que pueden pasar sin ocupar su PC, siendo estas diferencias menos elevadas (y, por tanto, mayor dificultad de pasar sin dicho dispositivo) para los hombres.

$\mathrm{La} \mathrm{H}_{6}$ trata sobre las diferencias que reportan hombres y mujeres en cuanto al sentimiento de culpa por estar largo tiempo utilizando los dispositivos tecnológicos. Al igual que en la hipótesis anterior, valores más elevados implican menor sentimiento de culpa. Los resultados de la prueba $t$ de Student para muestras independientes (Tabla 1) indican que la única diferencia importante se da en el caso de la consola de juegos: el hombre es quien más culpa reporta.

En cuanto a la $\mathrm{H}_{7}$, que trata el tema de la adicción a la tecnología, las pruebas de chi cuadrado arrojan los siguientes resultados. En la percepción 
de adicción, sí existen diferencias significativas $\left(\chi^{2}=40.88 ; \mathrm{p}=000\right)$ : entre los que se consideran adictos no las hay, pero sí entre quienes no se consideran (véase Figura 2). Por último, en torno a la pregunta de si regresaron alguna vez a casa, trabajo o lugar de estudios por haber olvidado su dispositivo móvil sin importar que estuvieran atrasados, las diferencias también son significativas $\left(\chi^{2}=15.86 ; \mathrm{p}=000\right)$ : las mujeres reportaron en mayor medida una respuesta negativa en comparación con los hombres (véase Figura 3).

Cabe destacar que al mostrar los resultados de las siete hipótesis que conforman este artículo, se debe tomar en cuenta que aquellos se limitan a la muestra de estudio, sin generalizar.

\section{Discusión}

Estudios previos han confirmado que la tecnología interfiere en la vida cotidiana de las personas (Roberts y David, 2016: 140). El presente artículo muestra que los hombres son quienes pasan más tiempo utilizando dispositivos tecnológicos y quienes reportan un mayor sentimiento de culpa. En cuanto al impulso para utilizar tecnología, hay una marcada diferencia en ambos géneros. Lo que más les impulsa es estar conectados con la familia, pero las mujeres reportan una puntuación mayor que los hombres. En todas las demás actividades se mantienen de manera similar los dos géneros. Cabe señalar que los hombres presentan mayores problemas por interferencia de la tecnología, sobre todo cuando se trata de conflictos en su casa.

Cuando se observa los lugares donde hay problemas por la interferencia de la tecnología (véase la Figura 4), tanto hombres y mujeres muestran similares tendencias en los mismos sitios, pero donde hay una diferencia significativa un poco más evidente para hombres que para mujeres son: en el trabajo, haciendo deporte y en tiendas o centros comerciales. Estos resultados están relacionados directamente con la corriente ciberfeminista, al evidenciar que en los usos de dispositivos tecnológicos no hay dominación patriarcal masculina, al igual que no existe brecha digital de género en el presente estudio; además, se confirman los datos de Hargittai y Walejko (2008: 252) respecto a la igualdad de género en el acceso de las TIC en ciertas actividades.

Al revisar las características del phubbing, se encontró que los hombres presentan mayores evidencias de ser phubbers; un resultado que confirma la existencia de phubbing en la muestra examinada y que se relaciona con los estudios de Roberts y David (2016: 140), acerca de la preferencia que se les da a los dispositivos tecnológicos por encima de las personas que están alrededor. Por ejemplo, en la presente investigación, para el caso del ocio, los 
hombres muestran en todos los dispositivos marcadas diferencias: Pc ( $t=-$ 7.42), SP $(t=-4.21)$, tablet $(t=-4.97)$, consola $(t=-11.20)$, en comparación con las mujeres (véase Tabla 1). Respecto a los tiempos de uso con el ocio, por ejemplo, consola de juegos, se constató que los hombres ocupan mayor tiempo utilizando este dispositivo para dicha actividad. Asimismo, al hacer una comparación con las investigaciones de Rideout et al. (2010: 21) y de Gómez et al. (2018: 71), se puede observar que se mantienen los mismos comportamientos en hombres y mujeres, aun cuando hay diferencia de edades entre los grupos examinados.

Por último, los resultados del estudio exponen la percepción de que no existen diferencias significativas entre quienes reconocen que, efectivamente, sufren una adicción a la tecnología; en tanto, entre quienes no aceptan tener esta adicción, sí se presentan diferencias importantes. La mayoría de las mujeres indicaron no ser adictas, pero aun así regresaron a casa por su dispositivo olvidado, sin importar que estuvieran atrasadas. En este caso, la investigación se basa en la propia percepción de los encuestados sobre: sus sentimientos de culpa, el tiempo de uso de los dispositivos, el tipo de problemas que han tenido por la interferencia de la tecnología, el hecho de regresar a casa por los dispositivos olvidados, tener gastos, entre otras cuestiones relacionadas con los estudios de González et al. (2018: 82).

\section{Limitaciones y futuros estudios}

Aunque una de las fortalezas es el alto número de participantes, como ya se ha dicho, la muestra ha sido compuesta principalmente por estudiantes de una comunidad académica de Quito, por lo cual es necesario ampliar la muestra hacia otros sectores.

Por otra parte, sería esencial ahondar en el tipo de motivaciones o razones que impulsan a hombres y mujeres a realizar diferentes actividades por varios periodos a través de los diversos dispositivos tecnológicos; también sería interesante determinar las prioridades e importancia que les dan a cada uno de estos. De igual manera, habría que profundizar en el tipo de problemas que tienen hombres y mujeres por la interferencia de la tecnología en áreas como: trabajo, familia y sociedad.

Por tanto, este estudio es un primer paso en el entendimiento de las TIC en la sociedad actual, cómo afectan a la vida cotidiana y las diferencias que existen debido al género. 


\section{Conclusiones}

Como resultado se puede señalar que, aunque no hay dominación patriarcal ni brecha digital de género -se puede evidenciar acceso igualitario entre géneros-, son los hombres quienes pasan más tiempo utilizando dispositivos tecnológicos y quienes reportan un mayor sentimiento de culpa frente al caso de las mujeres. Además, aunque tanto para hombres como para mujeres el estar conectados con la familia es lo que más los impulsa a ocupar dispositivos tecnológicos, las mujeres reportan una mayor puntuación que los hombres. En cuanto a la interferencia, los hombres presentan mayor cantidad de problemas por esto que las mujeres, sobre todo en el trabajo, haciendo deporte y en tiendas o centros comerciales. Es importante destacar que se confirma la presencia de phubbing, y son los hombres quienes presentan mayores evidencias de actuar como phubbers por prestar más atención a los dispositivos tecnológicos que a las personas a su alrededor. Y finalmente, al momento de reconocer si sufren o no de adicción a la tecnología, la percepción de los participantes en el presente estudio no evidencia diferencias significativas, aun cuando las mujeres, en la mayoría de los casos, han indicado que no son adictas, a pesar de haber regresado a su casa por su dispositivo olvidado, sin importar que estuvieran atrasadas.

\section{Referencias}

Cáceres, María Dolores et al. (2017), "Sociabilidad virtual: La interacción social en el ecosistema digital”, en Historia y Comunicación Social, vol. 22, núm. 1, España: Universidad Complutense. DOI: http://dx.doi.org/10.5209/HICS.5591.

Cantos, J. L. y Collado, C. C. (2017), "La brecha digital de género y la escasez de mujeres en las profesiones TIC”, en Panorama social, núm. 25, España: Funcas.

Castaño, Cecilia et al. (2010), "La brecha digital de género: amantes y distantes”, en Estudios de la Universidad Complutense, España: Ediciones Complutense. Disponible en: http://ateneu.xtec.cat/wikiform/wikiexport/_media/cursos/escola_inclusiva/cot1/ modul_1/amantesydistantes_0.pdf [02 de septiembre de 2017].

Castells, Manuel (1996), The rise of the network society, Reino Unido: Blackwell publishers.

Cotten, Sheila et al. (2009), "Old wine in a new technology or a new type of digital divide?", en New Media \& Society, núm. 11, vol. 7, Inglaterra: Sage.

Ensmenger, Nathan (2010), The computer boys take over: computers, programmers, and the politics of technical expertise, Reino Unido: MIT Press.

Gómez, María Claudia (2017), "Estudio exploratorio sobre hábitos y experiencias de consumo tecnológico de tweens guayaquileños por género", en Estudio cualitativo Bachelor's thesis, Universidad Casa Grande. Facultad de Comunicación Monica Herrera, Ecuador: Universidad Casa Grande. 
González, Aníbal et al. (2018), "Phubbing en las relaciones románticas: uso del celular, satisfacción en la pareja, bienestar psicológico y salud mental" en Interacciones, Revista de Avances en Psicología, vol. 4, núm. 2, Perú: Instituto Peruano de Orientación Psicológica.

Haigh, Alex (2013), “Stop phubbing”. Disponible en: http://stopphubbing.com/ [02 de septiembre de 2017].

Hargittai, Eszter y Walejko, Gina (2008), “The participation divide: Content creation and sharing in the digital age", en Information, Communication and Society, núm. 11, vol. 2, Reino Unido: Taylor \& Francis.

Isaacson, Walter (2014), The innovators, Estados Unidos: Simon and Schuster.

Naciones Unidas (1996), Informe de la Cuarta Conferencia Mundial sobre la Mujer, del 4 al 15 de septiembre de 1995, Estados Unidos: Naciones Unidas. Disponible en: http:// www.un. org/womenwatch/daw/beijing/pdf/Beijing\%20full\%20report\%20S.pdf [8 de junio de 2018].

Reverter, Sonia (2013), “Ciberfeminismo de virtual a político”, en Teknokultura, Revista de Cultura Digital y Movimientos Sociales, núm. 2, vol. 10, España: Ediciones Complutenses.

Rideout, Victoria et al. (2010), "Generation M2: Media in the lives of 8- to 18-Year-Olds", en Kaiser family foundation, Estados Unidos: Henry J. Kaiser Family Foundation. Disponible en: https://files.eric.ed.gov/fulltext/ED527859.pdf [02 de septiembre de 2017].

Roberts, James y David, Meredith (2016), "My life has become a major distraction from my cell phone: Partner phubbing and relationship satisfaction among romantic partners", en Computers in Human Behavior, núm. 54, Holanda: Elsevier.

Rodríguez, Amelia (2009), "Género y TIC. Hacia un nuevo modelo más equilibrado o la Sociedad de la Información a dos velocidades”, en Lecciones del Portal. Disponible en http://www.portalcomunicacion.com/uploads/pdf/52_esp.pdf [02 de septiembre de 2017].

Rodríguez, Rubén et al. (2012), "Adicción a las nuevas tecnologías de la información y la comunicación (ntics) y ansiedad en adolescentes", en International Journal of Developmental and Educational Psychology, núm. 1, vol. 1, España: Asociación Nacional de Psicología Evolutiva y Educativa de la Infancia, Adolescencia y Mayores.

Sanou, Brahima (2016), "ICT: facts \& figures 2016". Disponible en: https://www.itu.int/ en/ITU-D/Statistics/Documents/facts/ICTFactsFigures2016.pdf [02 de septiembre de 2017].

Sanz, Verónica (2006), "Las tecnologías de la información desde el punto de vista de género: posturas y propuestas desde el feminismo", en Isegoría, núm. 34, España: Consejo Superior de Investigaciones Científicas.

Treviño, Joel (2013), “El 'phubbing', otra amenaza para las relaciones interpersonales y laborales", en Abogados, México: Abogado digital. Disponible en: http:// www.lexinformatica.com/blog/el-phubbing-otra-amenaza-para-las-relacionesinterpersonales-y-laborales/ [02 de septiembre de 2017]. 


\section{Anexo}

\section{Tabla 1}

\section{Comparación de medias $(\mathrm{N}=3793)$}

\begin{tabular}{|c|c|c|c|}
\hline & \multicolumn{2}{|c|}{$\mathrm{M}$} & \multirow[t]{2}{*}{ Estadístico } \\
\hline & $\mathrm{H}$ & $\mathrm{M}$ & \\
\hline Periodicidad PC & 4.03 & 3.85 & $\mathrm{t}=-3.23(\mathrm{p}=.001)$ \\
\hline Periodicidad Smartphone (SP) & 4.06 & 3.68 & $\mathrm{t}=-5.12(\mathrm{p}=.000)$ \\
\hline Periodicidad tablet & 2.20 & 1.91 & $\mathrm{t}=-5.81(\mathrm{p}=.000)$ \\
\hline Periodicidad consola & 1.97 & 1.27 & $\mathrm{t}=-17.84(\mathrm{p}=.000)$ \\
\hline Buscar información & 3.41 & 3.43 & $\mathrm{t}=-., 45(\mathrm{p}=.654)$ \\
\hline Hacer compras en línea & 1.54 & 1.29 & $\mathrm{t}=8,03(\mathrm{p}=.000)$ \\
\hline Estudios & 3.57 & 3.51 & $\mathrm{t}=1,28(\mathrm{p}=.200)$ \\
\hline Redes sociales & 3.63 & 3.37 & $\mathrm{t}=4,55(\mathrm{p}=.000)$ \\
\hline Reproducir videos & 3.32 & 3.02 & $\mathrm{t}=5,70(\mathrm{p}=.000)$ \\
\hline Autoeducación & 3.40 & 3.26 & $\mathrm{t}=2,81(\mathrm{p}=.005)$ \\
\hline Juegos en red & 2.14 & 1.55 & $\mathrm{t}=13,74(\mathrm{p}=.000)$ \\
\hline Ocio & 2.47 & 1.95 & $\mathrm{t}=10,55(\mathrm{p}=.000)$ \\
\hline Servicios de comunicación & 3.01 & 2.75 & $\mathrm{t}=4,72(\mathrm{p}=.000)$ \\
\hline Trabajo & 3.19 & 2.82 & $\mathrm{t}=5.59(\mathrm{p}=.000)$ \\
\hline Buscar información con PC & 51.40 & 51.63 & $\mathrm{t}=.22(\mathrm{p}=.826)$ \\
\hline Buscar información con SP & 30.23 & 26.19 & $\mathrm{t}=-.97(\mathrm{p}=.000)$ \\
\hline Buscar información con tablet & 13.44 & 12.73 & $\mathrm{t}=-.85(\mathrm{p}=.392)$ \\
\hline Buscar información con consola & 5.47 & 2.48 & $\mathrm{t}=-6.60(\mathrm{p}=.000)$ \\
\hline Hacer compras en línea con PC & 10.03 & 5.94 & $\mathrm{t}=-5.90(\mathrm{p}=.000)$ \\
\hline Hacer compras en línea con SP & 7.46 & 4.45 & $\mathrm{t}=-5.34(\mathrm{p}=.000)$ \\
\hline Hacer compras en línea con tablet & 4.99 & 2.85 & $\mathrm{t}=-4.91(\mathrm{p}=.000)$ \\
\hline Hacer compras en línea con consola & 3.70 & 1.41 & $\mathrm{t}=-6.56(\mathrm{p}=.000)$ \\
\hline Estudios con PC & 40.70 & 36.91 & $\mathrm{t}=-3.43(\mathrm{p}=.001)$ \\
\hline Estudios con SP & 21.89 & 18.40 & $\mathrm{t}=-4.01(\mathrm{p}=.000)$ \\
\hline Estudios con tablet & 11.07 & 8.73 & $\mathrm{t}=-3.45(\mathrm{p}=.001)$ \\
\hline Estudios con consola & 3.75 & 2.11 & $\mathrm{t}=-4.37(\mathrm{p}=.000)$ \\
\hline Redes sociales con PC & 34.43 & 29.46 & $\mathrm{t}=-4.78(\mathrm{p}=.000)$ \\
\hline Redes sociales con SP & 35.95 & 30.74 & $\mathrm{t}=-4.64(\mathrm{p}=.000)$ \\
\hline Redes sociales con Tablet & 12.14 & 10.24 & $\mathrm{t}=-2.53(\mathrm{p}=.011)$ \\
\hline Redes sociales con consola & 5.09 & 2.48 & $\mathrm{t}=-6.05(\mathrm{p}=.000)$ \\
\hline Reproducir videos con PC & 36.18 & 29.76 & $\mathrm{t}=-5.90(\mathrm{p}=.000)$ \\
\hline Reproducir videos con SP & 24.81 & 20.81 & $\mathrm{t}=-4.11(\mathrm{p}=.000)$ \\
\hline Reproducir videos con tablet & 11.47 & 9.60 & $\mathrm{t}=-2.54(\mathrm{p}=.011)$ \\
\hline Reproducir videos con consola & 7.33 & 2.80 & $\mathrm{t}=-8.85(\mathrm{p}=.000)$ \\
\hline Autoeducación con PC & 33.92 & 30.56 & $\mathrm{t}=-3.11(\mathrm{p}=.002)$ \\
\hline
\end{tabular}


Cristian Moisés Villafuerte-Garzón y María Vera-Perea. Phubbing y género en un sector académico en Quito: Uso, abuso e interferencia de la tecnología

\begin{tabular}{|c|c|c|c|}
\hline & \multicolumn{2}{|c|}{ M } & \multirow[t]{2}{*}{ Estadístico } \\
\hline & $\mathrm{H}$ & $\mathrm{M}$ & \\
\hline Autoeducación con SP & 19.84 & 16.35 & $\mathrm{t}=-4.70(\mathrm{p}=.000)$ \\
\hline Autoeducación con tablet & 9.60 & 7.76 & $\mathrm{t}=-2.85(\mathrm{p}=.004)$ \\
\hline Autoeducación con consola & 3.42 & 1.81 & $\mathrm{t}=-4.55(\mathrm{p}=.000)$ \\
\hline Juegos en red con PC & 15.20 & 8.63 & $\mathrm{t}=-8.28(\mathrm{p}=.000)$ \\
\hline Juegos en red con SP & 11.06 & 8.62 & $\mathrm{t}=-3.37(\mathrm{p}=.001)$ \\
\hline Juegos en red con tablet & 6.93 & 4.71 & $\mathrm{t}=-4.02(\mathrm{p}=.000)$ \\
\hline Juegos en red con consola & 16.16 & 3.59 & $\mathrm{t}=-16.48(\mathrm{p}=.000)$ \\
\hline Ocio con PC & 18.02 & 11.98 & $\mathrm{t}=-7.42(\mathrm{p}=.000)$ \\
\hline Ocio con SP & 15.38 & 11.96 & $\mathrm{t}=-4.21(\mathrm{p}=.000)$ \\
\hline Ocio con tablet & 7.38 & 4.70 & $\mathrm{t}=-4.97(\mathrm{p}=.000)$ \\
\hline Ocio con consola & 9.67 & 2.90 & $\mathrm{t}=-11.20(\mathrm{p}=.000)$ \\
\hline Servicios de comunicación con PC & 24.78 & 19.84 & $\mathrm{t}=-5.04(\mathrm{p}=.000)$ \\
\hline Servicios de comunicación con SP & 23.08 & 18.42 & $\mathrm{t}=-4.63(\mathrm{p}=.000)$ \\
\hline Servicios de comunicación con tablet & 7.96 & 6.30 & $\mathrm{t}=-2.73(\mathrm{p}=.006)$ \\
\hline Servicios de comunicación con consola & 3.92 & 1.63 & $\mathrm{t}=-6.34(\mathrm{p}=.000)$ \\
\hline Trabajo con PC & 28.01 & 23.52 & $\mathrm{t}=-3.93(\mathrm{p}=.000)$ \\
\hline Trabajo con SP & 16.31 & 10.35 & $\mathrm{t}=-7.44(\mathrm{p}=.000)$ \\
\hline Trabajo con tablet & 7.39 & 4.66 & $\mathrm{t}=-4.92(\mathrm{p}=.000)$ \\
\hline Trabajo con consola & 3.09 & 1.21 & $\mathrm{t}=-5.84(\mathrm{p}=.000)$ \\
\hline Problemas de interferencia en la casa & 1.92 & 1.85 & $\mathrm{t}=-2.31(\mathrm{p}=.021)$ \\
\hline Problemas de interferencia en el trabajo & 1.59 & 1.48 & $\mathrm{t}=-4.10(\mathrm{p}=.000)$ \\
\hline Problemas de interferencia en el lugar de estudios & 1.82 & 1.74 & $\mathrm{t}=-2.86(\mathrm{p}=.004)$ \\
\hline Problemas de interferencia en medios de transporte & 1.77 & 1.62 & $\mathrm{t}=-4.89(\mathrm{p}=.004)$ \\
\hline Problemas de interferencia haciendo deporte & 1.60 & 1.44 & $\mathrm{t}=-5.63(\mathrm{p}=.000)$ \\
\hline Problemas de interferencia en centros comerciales & 1.74 & 1.60 & $\mathrm{t}=-4.45(\mathrm{p}=.000)$ \\
\hline Problemas de interferencia en reuniones sociales & 1.78 & 1.68 & $\mathrm{t}=-3.29(\mathrm{p}=.001)$ \\
\hline Tiempo sin usar el PC & 5.64 & 5.96 & $\mathrm{t}=2.89(\mathrm{p}=.004)$ \\
\hline Tiempo sin usar el SP & 5.14 & 5.06 & $\mathrm{t}=-.71(\mathrm{p}=.477)$ \\
\hline Tiempo sin usar la tablet & 5.75 & 5.83 & $\mathrm{t}=.58(\mathrm{p}=.562)$ \\
\hline Tiempo sin usar la consola & 5.89 & 5.82 & $\mathrm{t}=-.50(\mathrm{p}=.619)$ \\
\hline Reporte de culpa por uso de PC & 3.47 & 3.46 & $\mathrm{t}=-.17(\mathrm{p}=.863)$ \\
\hline Reporte de culpa por uso de SP & 3.79 & 3.87 & $\mathrm{t}=1.27(\mathrm{p}=.206)$ \\
\hline Reporte de culpa por uso de tablet & 4.39 & 4.50 & $\mathrm{t}=1.77(\mathrm{p}=.076)$ \\
\hline Reporte de culpa por uso de consola & 4.50 & 4.93 & $\mathrm{t}=7.14(\mathrm{p}=.000)$ \\
\hline
\end{tabular}

Fuente: Elaboración propia. 


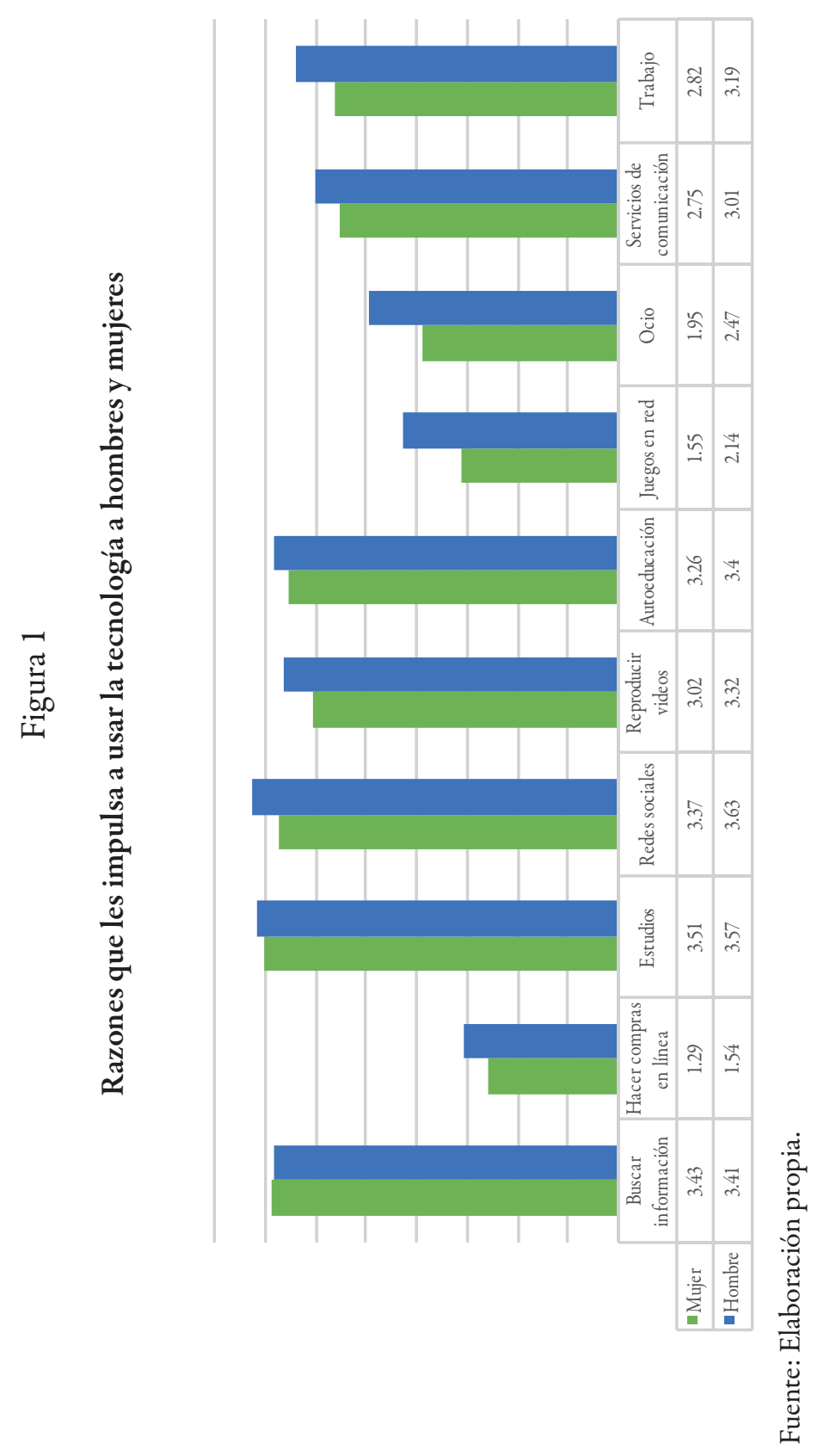


Cristian Moisés Villafuerte-Garzón y María Vera-Perea. Phubbing y género en un sector académico en Quito: Uso, abuso e interferencia de la tecnología

Figura 2

\section{Percepción de adicción}

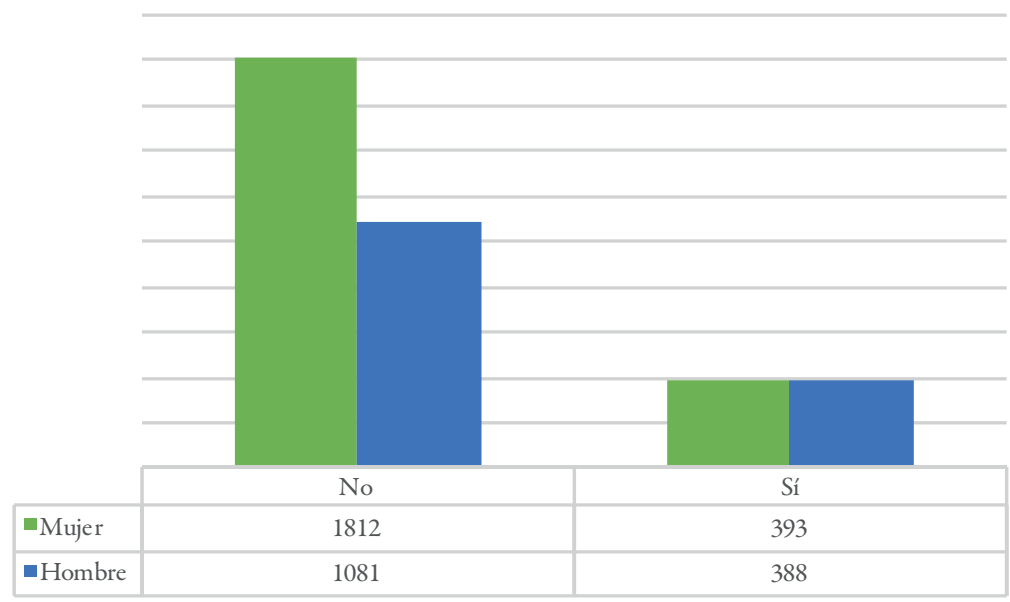

Fuente: Elaboración propia.

Figura 3

Regresar por haberse olvidado el dispositivo

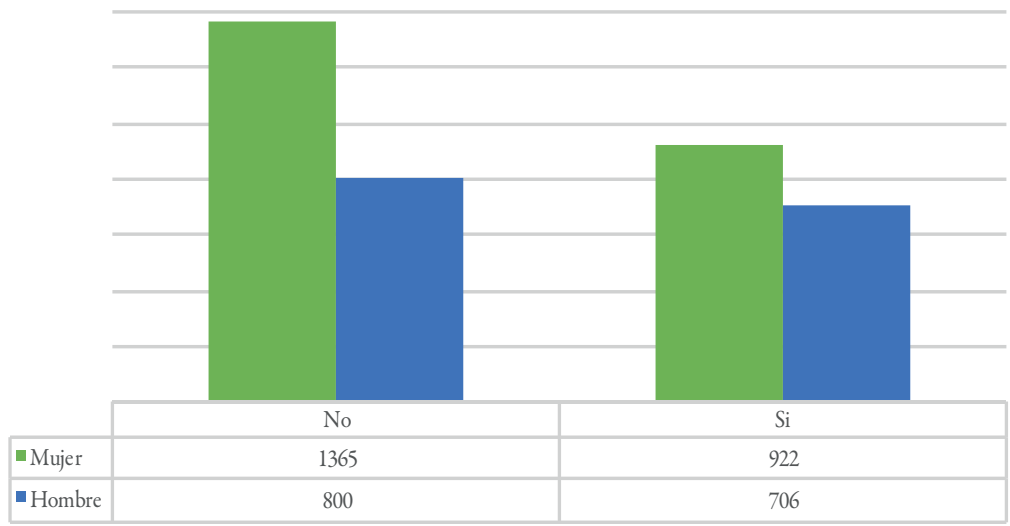

Fuente: Elaboración propia. 


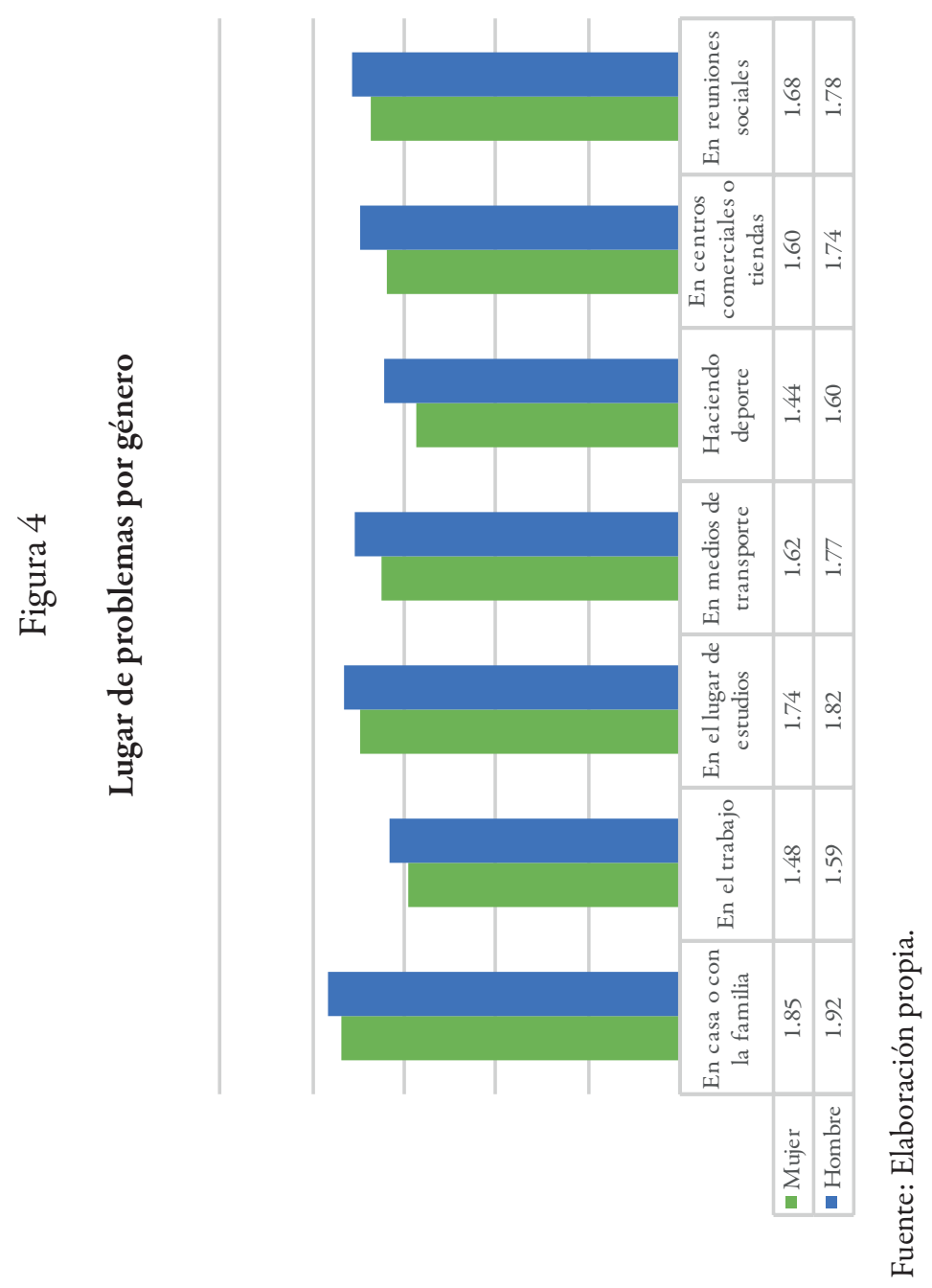


Cristian Moisés Villafuerte-Garzón. Coordinador General Académico en el Tecnológico Superior Cordillera, analista de Sistemas por el Tecnológico Superior Cordillera, Quito, Ecuador, Diplomado Superior en Educación y Nueva Tecnologías por FLACSO, Buenos Aires, Argentina. Líneas de investigación: tendencias de consumo, educación, nuevas tecnologías. Publicaciones recientes: Villafuerte-Garzón, Cristian Moisés (2018), "Ocio digital y los problemas del uso excesivo de dispositivos tecnológicos”, en Revista Polo del Conocimiento, Edición Núm. 22. Vol. 3, No 8. Agosto 2018, pp. 471-485. ISSN: 2550 - 682X DOI: 10.23857/casedelpo.2018.3.8.agosto.471-485 Ecuador: Casa Editora del Conocimiento; Villafuerte-Garzón, Cristian Moisés (2017), "Educación y nuevas tecnologías, una perspectiva del uso estudiantil en educación superior", en Revista Yura: Relaciones Internacionales, vol. 12, octubre-diciembre, Ecuador: Red de Investigación sobre Complejidad en América Latina y el Caribe.

María Vera-Perea. PhD en Psicología del Trabajo y las Organizaciones por la Universitat Jaume I. Profesora en la Universidad Especialidades Espíritu Santo (Facultad de Artes Liberales, Escuela de Psicología). Líneas de investigación: autoeficacia, bienestar, engagement, resiliencia. Publicaciones recientes: Vera, M., Rodríguez-Sánchez, A. y Salanova, M. (2017), "May the force be with you: Looking for resources that build team resilience", en Journal of Workplace Behavioral Health, Inglaterra: Taylor \& Francis, DOI:10.1080/1 5555240.2017.1329629; Cortés, J. A., Vera, M. y Cifre, E. (2017), “Relación entre la inteligencia emocional del líder y el flow de sus trabajadores/as", en Revista Interamericana de Psicología Ocupacional, vol. 34, núm. 2, Colombia: Centro de Investigación en Comportamiento Organizacional. 